\title{
KETOROLAC ENTERIC MATRIX PELLETS PRODUCED BY EXTRUSION / SPHERONIZATION
}

\author{
Gamal M. Mahrous* \\ Department of Pharmaceutics, Faculty of Pharmacy, King Saud University, Riyadh, Kingdom \\ of Saudi Arabia
}

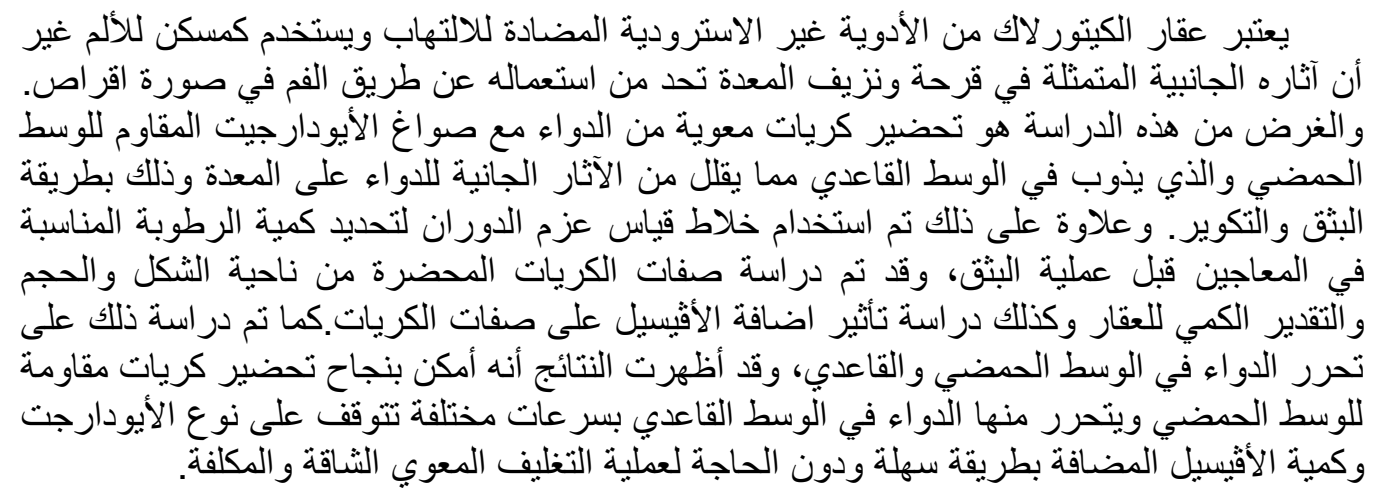

Ketorolac is a non-steroidal anti-inflammatory drug used in the treatment of pain. However, it has side effects including gastrointestinal (GI) irritation when administered orally. The oral administration of conventional oral dosage forms of ketorolac can cause serious gastrointestinal side effects and gastric irritation due to dose dumping. The objective of this study was to prepare enteric matrix pellets of the drug that lower the risk of side effects by the formulation of Eudragit / microcrystalline cellulose (Avicel PH 101) pellets using extrusion / spheronization technique. Moreover, mixer torque rheometer was used to quantitatively determine the suitable moisture content in the pastes before the extrusion process. The produced pellets were characterized for their ketorolac content, particle size, shape and dissolution profile. Studies on the effect of additives (Avicel and PVP) on Eudragit rheological properties revealed that the magnitude of torque lowered as the Avicel ratio increased, a fact that reflected on the roundness of the produced pellets. The release of the drug is controlled by the Eudragit/ Avicel ratio. In some formulations, in acidic medium, the release of the drug was less than 10\% after 2 hrs, while it is completely released within 60-120 min after changing dissolution medium to phosphate buffer ( $p H$ 6.8).

In conclusion, the manufactured pellets delayed drug release rates as well as it successfully passed the USP requirements for enteric delayed release articles, which might be beneficial in lowering the risk of side effects as an advantage of the pellets as drug delivery system without the need for tedious enteric coating process.

\section{INTRODUCTION}

Ketorolac is a non-steroidal antiinflammatory drug with potent analgesic activity. It is used in the water soluble salt form Ketorolac tromethamine (KT), to decrease gastrointestinal side effects that produced by the oral tablets.

Multiple-unit dosage forms have several advantages compared with single-unit dosage forms including more stable plasma profiles and little risk of local side effects ${ }^{1}$. Among the various types of multiple-unit dosage forms, pellets have attracted more attention due to their unique clinical and technical advantages. Pellets are defined as spherical, free-flowing granules with a narrow size distribution, typically varying between 500 and $2000 \mu$ for pharmaceutical applications ${ }^{2}$. The interest in pellets as dosage forms, filled into hard gelatin capsules or compressed into disintegrating 
tablets, has been increasing continuously. Pellets as a drug delivery system offer therapeutic advantages such as less irritation of the gastro-intestinal tract and a lowered risk of side effects due to dose dumping ${ }^{3}$.

Extrusion and spheronization is currently one of the techniques used to produce pharmaceutical pellets. With each production technique, pellets with specific characteristics are obtained. The preparation of spherical granules or pellets by extrusion and spheronization is now a more established method because of its advantages over the other methods ${ }^{2 \& 4}$. Enteric or sustained release pellets can either be of the matrix type where the drug is embedded in the release-controlling matrix, or of the reservoir type. The drugcontaining core of reservoir-type pellets is traditionally produced by wet-mass extrusion/spheronization or by layering of the drug onto non-pareils. A functional coating is then applied to the pellets in a subsequent step. Matrix systems may be produced by wet-mass extrusion and spheronization, melt granulation or hot-melt extrusion using release-controlling carriers ${ }^{5}$. The preparation of enteric matrix systems by hot-melt extrusion has been reported. Andrews and co-workers demonstrated that melt extruded tablets containing 5-aminosalicylic acid and Eudragit L100-55 released less than 5\% drug over $2 \mathrm{hrs}$ in acid medium ${ }^{6}$. Yang and co-workers prepared enteric matrix tablets releasing less than $3 \%$ drug by cutting as well as direct compression of comminuted extrudates when using Eudragit L100 as the matrix former ${ }^{7}$. Melt extruded Eudragit S100 tablets for the colonic delivery of 5-aminosalicylic acid also showed excellent gastric protection ${ }^{8}$. As a drug delivery system the enteric matrix pellets ensure less irritation of the gastro-intestinal tract and a lower risk of side effects ${ }^{9 \& 10}$.

In this study, Eudragit S100 (EUS) and Eudragit L100-55 (EUL) were used to prepare ketorolac enteric matrix systems by wet-mass extrusion and spheronization.

\section{MATERIALS AND METHODS}

Table 1: Composition of the prepared pellets.

\section{Materials}

Ketorolac tromehamine was purchased from Sigma Chemicals Co., USA. Eudragit ${ }^{\circledR}$ L100-55 and Eudragit ${ }^{\circledR}$ S100 were gifts from Rohm Pharma GmbH (Darmstadt, Germany). Polyvinylpyrrolidone (PVPK90) was supplied by Fluka (Switzerland). Microcrystalline cellulose (Avicel® PH101) was purchased from Serva Feinbiochemica (Heidelberg, Germany). All other materials and solvents used are of reagent or analytical grade and they were used without further purification.

\section{Methods \\ Wet massing studies using a mixer torque rheometer}

The mixer torque rheometer used in the present study consists of a $135-\mathrm{ml}$ capacity stainless steel bowl equipped with two mixing blades with rotational speed ranging between 20-150 rpm (MTR-3, Caleva, and Dorset, England). Depending on the bulk density, a sample of $15-20 \mathrm{~g}$ of dry powder material is sufficient to cover the mixer blades. The torque is measured directly on the mixer bowl with the help of a torque arm connected from the main body of the mixer to a calibrated load transducer. The data acquisition and analyses were carried out by a personal computer using data acquisition system and software package supplied by the equipment manufacturer.

Drug substance and excipients (Table 1) were mixed in turbula mixer (type S27, Erweka, Apparatebau, Germany). A 15 gram sample of this dry blend was utilized in these studies. Five millilitres of granulating fluid (deionized. water) were added in multiply additions over 4 wet massing intervals. Each wet massing interval consisted of a one minute mixing period and a 20 -second data logging (collection) period with the MTR operating at $50 \mathrm{rpm}$. Mean line torque was monitored during the granulation process.

\section{Preparation of Ketorolac (KT)-loaded pellets}

Pellets were prepared from those wet masses showing the highest mean torque. Formulations (Table 1) were mixed in turbula mixer for $5 \mathrm{~min}$ (type S27, Erweka, Apparatebau, Germany). The powder mixture

$$
\begin{array}{|l|l}
\text { Ingredient } & \text { Composition }(\% \mathrm{w} / \mathrm{w}) \text { of the prepared pellets }
\end{array}
$$




\begin{tabular}{||l|c|c|c|c|c|c|c|c|c||}
\hline & F1 & F2 & F3 & F4 & F5 & F6 & F7 & F8 & F9 \\
\hline Ketorolac & 5 & 5 & 5 & 5 & 5 & 5 & 5 & 5 & 5 \\
\hline EU S100 & - & 93 & 88 & 83 & 73 & - & - & - & - \\
\hline EU L100- 55 & - & - & - & - & - & 93 & 88 & 83 & 73 \\
\hline PVP K90 & 2 & 2 & 2 & 2 & 2 & 2 & 2 & 2 & 2 \\
\hline Avicel pH101 & 93 & - & 5 & 10 & 20 & - & 5 & 10 & 20 \\
\hline
\end{tabular}

was wetted with the calculated amount of water depending on the composition of the formulation. Next, the resulting wet mass was extruded at a speed of $90 \mathrm{rpm}$ (Mini Screw Extruder, Model MSE1014, Caleva, Dorset, England), through $1.6 \mathrm{~mm}$ diameter die. Spheronization was performed in a spheronizer (Model 120, Caleva, and Dorset, England) with a rotating plate of regular cross-hatch geometry, at a speed of $700 \mathrm{rpm}$, for $5 \mathrm{~min}$. Pellets were then dried on a tray in a hot oven at $50^{\circ} \mathrm{C}$ for $12 \mathrm{hrs}$.

\section{Determination of KT content in the prepared pellets}

Ketorolac (KT) content of the prepared pellets was determined spectrophotometrically (UV-9100 Spectrophotometer, Beijing Rayleigh Analytical Instruments Corp., China) at $320 \mathrm{~nm}$ in triplicate. Ketorolac loaded pellets were crushed in a porcelain mortar and an amount equivalent to $10 \mathrm{mg}$ of $\mathrm{KT}$ was dispersed in $50 \mathrm{ml}$ phosphate buffer $(\mathrm{pH}=7.4)$ under sonication. The solution was filtered and KT amount was measured spectrophotometrically at $320 \mathrm{~nm}$ after appropriate dilution.

\section{Sieve analysis and yield of pellets}

The pellets were sieved using nest of standard sieves $(2000,1700,1400,1180,1000$, and $850 \mu$ ) shaken for $10 \mathrm{~min}$ on a sieve shaker (Retsch-Germany). The pellets retained on each sieve was weighed and the obtained data were used to calculate the average particle size. The size range of 1000-2000 um was considered appropriate and the weight of pellets in this range was reported as yield of pellets.

\section{Morphological analysis of the prepared pellets}

The morphological characteristics of the prepared pellets were observed by scanning electron microscopy (SEM). The samples were sputter-coated with thin gold palladium layer under an argon atmosphere using a gold sputter module in a high-vacuum evaporator. The coated samples were then scanned and photomicrographs were taken with an SEM (Jeol JSM-1600, Tokyo, Japan).

\section{Differential scanning calorimetry (DSC)}

Samples (3-5 mg) were hermetically sealed in aluminium pans and heated at a constant rate of $10^{\circ} \mathrm{C} / \mathrm{min}$, over a temperature range of $25^{\circ} \mathrm{C}$ to $250^{\circ} \mathrm{C}$. Thermograms of the samples were obtained using differential scanning calorimetry (DSC-60, Shimadzu, Japan). Thermal analysis data were recorded using a TA 50I PC system with Shimadzu software programs. Indium standard was used to calibrate the DSC temperature and enthalpy scale. N2 was used as a purging gas at rate of $30 \mathrm{ml} / \mathrm{min}$.

\section{In-vitro release studies of the prepared pellets}

The release measurements were performed using USP dissolution apparatus I at $50 \mathrm{rpm}$ (Caleva Ltd., Model 85T, Philips, Maidstone, UK) using a continuous automated monitoring system. This system consists of an IBM computer PK 8620 series and PU 8605/60 dissolution test software, Philips VIS/UV/NIR single beam eight cells spectrophotometer model PU 8620, and Watson-Marlow peristaltic pump. In each flask, $750 \mathrm{ml}$ of $0.1 \mathrm{~N}$ $\mathrm{HCl}$ was placed and the temperature was maintained at $37 \pm 0.5^{\circ} \mathrm{C}$. An accurately weighed amount (equivalent to $10 \mathrm{mg} \mathrm{KT}$ ) of the prepared pellets (filled in to a hard gelatin capsule) was placed in each of the six baskets that assembled and immersed in to each flask, and the test was run for two hours, and then the $\mathrm{pH}$ was changed to 6.8 by adding $250 \mathrm{ml}$ of 0.2 $\mathrm{M}$ trisodium phosphate or to $\mathrm{pH} 7.2$ by adding $250 \mathrm{ml}$ of $0.24 \mathrm{M}$ trisodium phosphate. The test 
continued for extra three hours and absorbance was recorded automatically at $320 \mathrm{~nm}$. The percentage of drug released was calculated as a function of time. The data presented are the mean of the six readings.

\section{RESULTS AND DISCUSSION}

\section{Wet massing studies using a mixer torque rheometer}

For the Eudragit ${ }^{\circledR}$ systems, the mean line torque initially showed an increase and then a gradual decrease with an increase in the amount of wet massing liquid (Figs. 1,2). According to Parker and Rowe ${ }^{11}$, the degree of liquid spreading and wetting as well as the substrate binder interaction will determine the relative positions of the peak values of mean line torque. It could be observed that an increase in the mean torque with the increase in the wet massing liquid level with a sharp peak followed by a drop in the torque as overwetting of the powder mass occurred. Moreover, the magnitude of torque for the Eudragit ${ }^{\circledR}$ systems was not as high in the present studies as that reported for other pelletization aids such as Avicel where water alone was used as the wet-massing liquid. This may possibly be due to better interaction of Avicel with water which has been discussed thoroughly in the literature ${ }^{12 \& 13}$. Extensive hydrogen bonding, large surface area and mechanical interlocking of the irregular particles have been suggested to be the factors responsible for the excellent binding property of Avicel $^{14}$. The hydroxyl groups in the cellulose chains form intra- and intermolecular hydrogen bonds and are likely hydrogen bonded with water molecules ${ }^{15}$. Therefore, Avicel was used in this study as a pelletization aid at three different concentrations (5, 10 and $20 \% \mathrm{w} / \mathrm{w})$. Also, in all formulations PVP was used as dry binder at $2 \% \mathrm{w} / \mathrm{w}$ concentration of the total powder blend to aid the binding properties of Eudragit. The use of PVP to aid the binding properties of Eudragit is previously mentioned in literature ${ }^{16 \& 17}$.

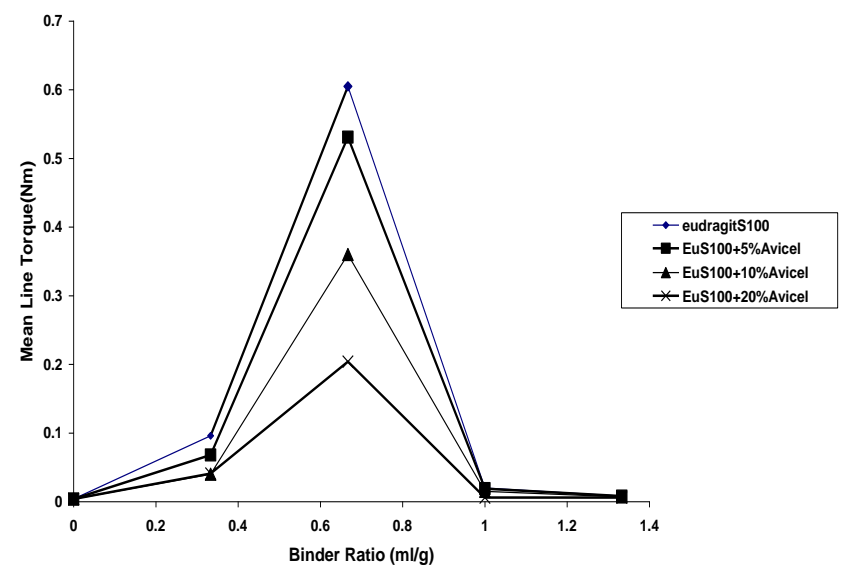

Fig. 1: Effect of water on the mean torque for Eudragit S100/ Avicel® pH101 blends.

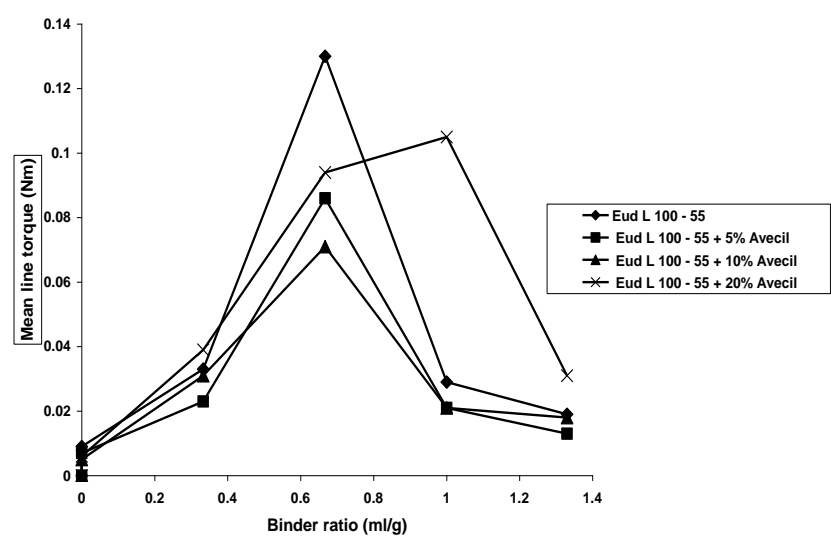

Fig. 2: Effect of water on the mean torque for Eudragit L100-55/Avicel® pH101 blends.

Figures (1\&2) illustrate the rheological profile obtained with Eudragit S100 and Eudragit L100-55 using different concentrations of Avicel. It can be seen that increasing concentration of Avicel lead to a decrease in the mean torque without change in the wet massing liquid ratio, with the exception of the 20\% Avicel / Eudragit L100-55 which showed an increase in the wet massing liquid ratio (Fig. 2). This increase in the binder ratio could be attributed to the slower change from the pendular to the funicular states due to increased water absorption capacity of the blend. The maximum consistencies (the peak torques) of the wet granules were obtained using $0.67 \mathrm{ml}$ of water/g of dry blend for all formulations except formula No. (9) which needs $1.2 \mathrm{ml} / \mathrm{g}$.

Size of the pellets

The results showed that all formulations produced acceptable yield at desired range i.e. 
$70 \%$ of the pellets were in the size range of $1-2$ $\mathrm{mm}$. The average particle size for each of the prepared formulae is listed in Table (2).

Table 2: Ketorolac content, pellet yield (1-2 $\mathrm{mm}$ ), and average particle size (D) of the produced pellets.

\begin{tabular}{||c|c|c|c||}
\hline $\begin{array}{c}\text { Formula } \\
\text { No. }\end{array}$ & $\begin{array}{c}\text { KT Content } \\
(\% \mathrm{w} / \mathrm{w})\end{array}$ & $\begin{array}{c}\text { Pellet yield } \\
(\% \mathrm{w} / \mathrm{w})\end{array}$ & $\mathrm{D}(\mu)$ \\
\hline F1 & $5.29 \pm 0.20$ & 85.5 & 1754 \\
\hline F2 & $4.89 \pm 0.31$ & 70.6 & 1480 \\
\hline F3 & $5.14 \pm 0.31$ & 72.4 & 1590 \\
\hline F4 & $4.93 \pm 0.10$ & 71.2 & 1605 \\
\hline F5 & $5.00 \pm 0.30$ & 73.8 & 1650 \\
\hline F6 & $5.04 \pm 0.07$ & 70.3 & 1492 \\
\hline F7 & $4.96 \pm 0.19$ & 73.4 & 1657 \\
\hline F8 & $5.14 \pm 0.08$ & 75.1 & 1720 \\
\hline F9 & $5.21 \pm 0.10$ & 76.2 & 1740 \\
\hline
\end{tabular}

\section{Drug content}

The drug content data of 5\% KT-loaded pellets are listed in Table (2). It is clearly evident that drug content is close to the theoretical value. These results can suggest obtaining uniform mixture of drug particles with the additives even after extrusion and spheronization.

\section{Morphological analysis}

Scanning electron micrographs of KT pellets containing $10 \%$ Avicel concentration (F4) are displayed in Figure (3). The resulting pellets were spherical and intact in shape. The pellets showed slightly rough surfaces. The images of the interior of the pellets revealed similar textures.

\section{Differential scanning calorimetry (DSC)}

The thermal behaviour of KT alone and as well as its physical mixtures with the used polymers are shown in Figure (4). The DSC traces of pure KT shows an endothermic peak at $160.26^{\circ} \mathrm{C}$ which is due to the melting of the drug. Avicel has no detected peak. The physical mixtures of drug with Avicel, EUS and EUL show endothermic peaks at the same position with less intensity (Fig. 4A,B). The weakness of the endothermic peak of KT of the physical mixtures in the presence of Avicel, EUS and EUL is attributed to the dilution effect of the polymers. These results indicate the compatibility of the used additives with the drug.
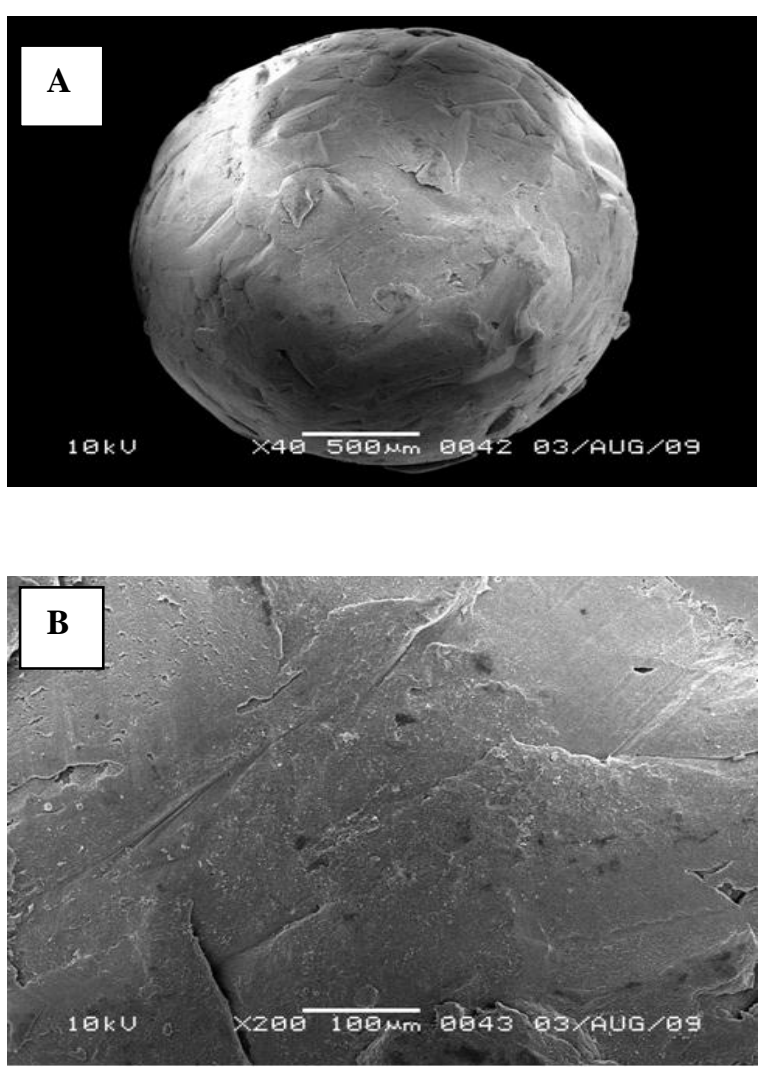

Fig. 3: Scanning electron micrographs of the pellets (A) and their surfaces (B) prepared form Eudragit S100/ Avicel® pH101 blend (F4).

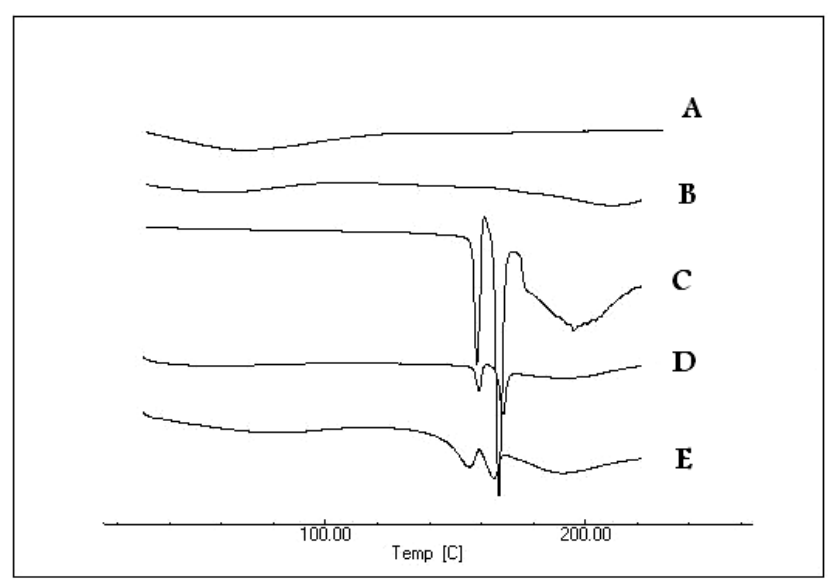

Fig. 4A: DSC curves of: A: Avicel, B: Eudragit S100, C: Ketorolac (KT), D: KT-Avicel, E: KT-Eudragit S100. 


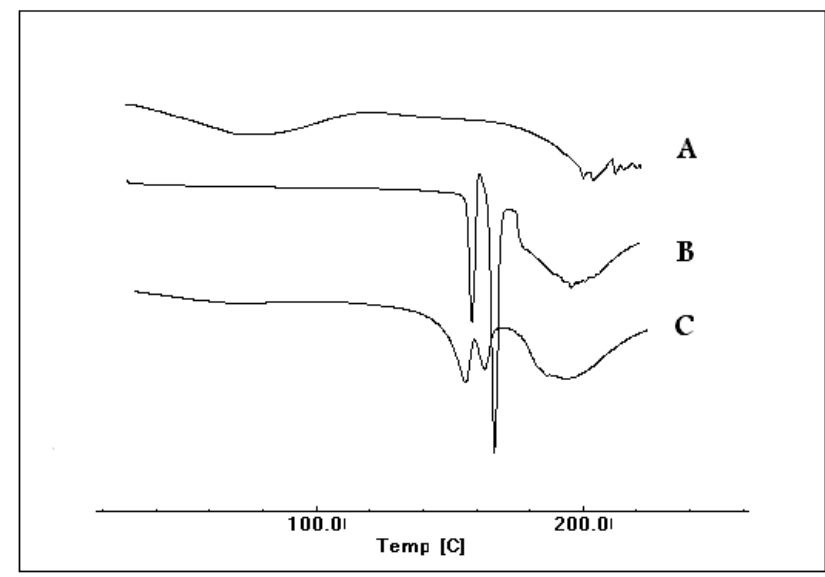

Fig. 4B: DSC curves of: A: Eudragit L100-55, B: Ketorolac (KT), C: KT-Eudragit L100-55.

\section{Drug release studies}

Drug release studies were performed according to the USP 24 criteria for enteric dosage forms. The pharmacopoeia stated that the enteric dosage form should not release more than $10 \%$ of the labeled amount in the acid stage $(0.1 \mathrm{~N} \mathrm{HCl}$ for $2 \mathrm{hrs})$. After $\mathrm{pH}$ changed to 6.8 , the dosage form should release most of the drug within the time stated in the monograph, (usually from $45 \mathrm{~min}$ to $90 \mathrm{~min})^{18}$. This criteria was applied to formulae containing EUL, (which dissolve at $\mathrm{pH}$ 5.5). However, for EUS formulations, extra drug release experiment was done, through which the $\mathrm{pH}$ was changed to 7.2 instead of 6.8 , as EUS dissolves at $\mathrm{pH}$ 7.0. KT release profiles of the extruded pellets are shown in Figures (5-7). The dissolution properties demonstrated the $\mathrm{pH}$ - dependent release mechanism of the formulations. Addition of Avicel causes decrease in drug release in all formulations. This could be attributed to the ability of Avicel as a water insoluble material to reduce the No. of pores in the matrix structure and the total pore surface area. Similar findings were previously reported ${ }^{17,19 \& 20}$. Figure (5) shows the release of ketorolac from formulations contain EUS (F2- F5), in addition to pellets made of Avicel only for comparison (F1). It is clear from the results that F1 and F2 (which contains EUS only) show high release in acid phase $(>10 \%)$. However, addition of Avicel to Eudragit lead to decrease in drug release from EUS formulae. The formula contains $20 \%$ Avicel (F5) showed sufficient acid resistance (9.5\% of KT was released after $2 \mathrm{hrs}$ ), while formulae contain 5\% \& $10 \%$ Avicel (F3\&F4) were not enough $(>10 \%$ release after $2 \mathrm{hrs})$. After $\mathrm{pH}$ change to 6.8 , as expected, $\mathrm{F} 1$ which contains Avicel only, complete dissolution is achieved after $1 \mathrm{hr}$ of the $\mathrm{pH}$ change. F2, which contains EUS only, showed about 80\% KT release by the end of run.

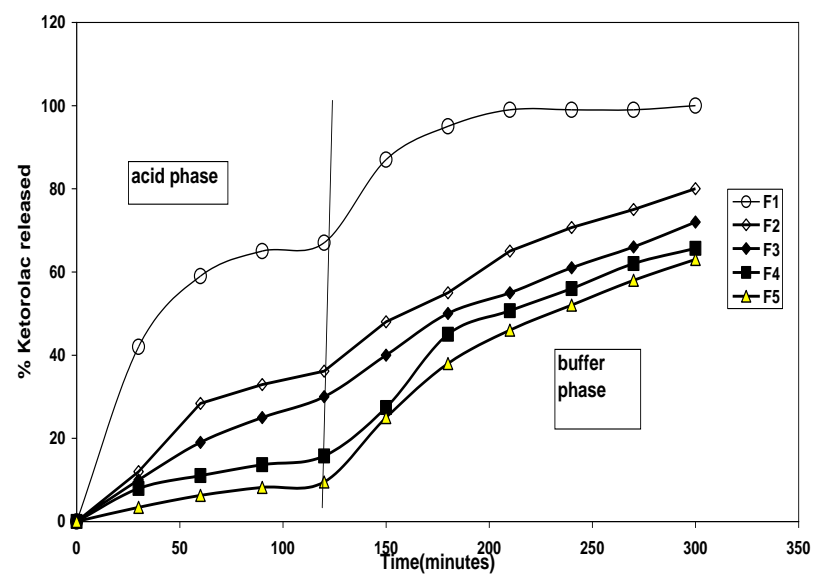

Fig. 5: Ketorolac release profiles from Eudragit $\mathrm{S} 100$ pellets in $0.1 \mathrm{~N} \mathrm{HCl}$, then in phosphate buffer at $\mathrm{pH} 6.8$, at $37^{\circ} \mathrm{C}$.

Figure (6) shows the release of $\mathrm{K} \mathrm{T}$ from formulations contain EUS at $\mathrm{pH} 1.2$ for $2 \mathrm{~h}$ then at $\mathrm{pH} 7.2$ for extra $3 \mathrm{hrs}$. It is obvious that the release rate increased in the phosphate buffer phase. This is due to the solubility of EUS at this pH. F5 is the only formula that passed the USP limits in the acid phase and showed KT release of about $80 \%$ by the end of test.

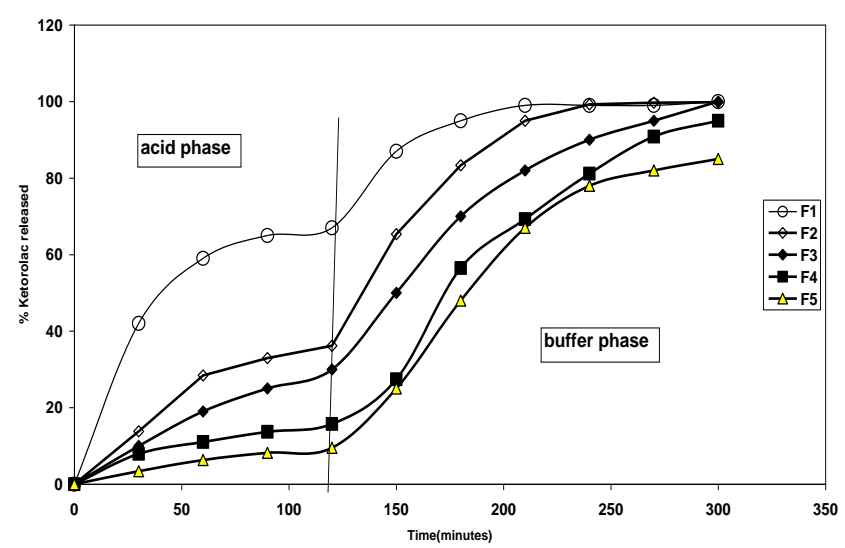

Fig. 6: Ketorolac release profiles from Eudragit S100 pellets in $0.1 \mathrm{~N} \mathrm{HCl}$, then in phosphate buffer at $\mathrm{pH} 7.2$, at $37^{\circ} \mathrm{C}$. 
Figure (7) shows the release of KT from formulations contains EUL at $\mathrm{pH} 1.2$ for $2 \mathrm{hrs}$ then at pH 6.8 for extra 3 hrs. It could be seen from the results that EUL formulation showed higher acid resistance than those containing EUS. This could be attributed to the lower acid solubility of EUL than EUS. Formulation contains 5\% Avicel only (F7) showed excellent acid resistance $(7.1 \%$ of KT was released after $2 \mathrm{hrs}$ ), and in the mean time, it showed faster release in buffer phase $(\mathrm{pH} \mathrm{6.8)}$ than the other test formulations. About $80 \%$ of the drug was released after one hour of $\mathrm{pH}$ change. Such formula could be a suitable enteric dosage form for ketorolac.

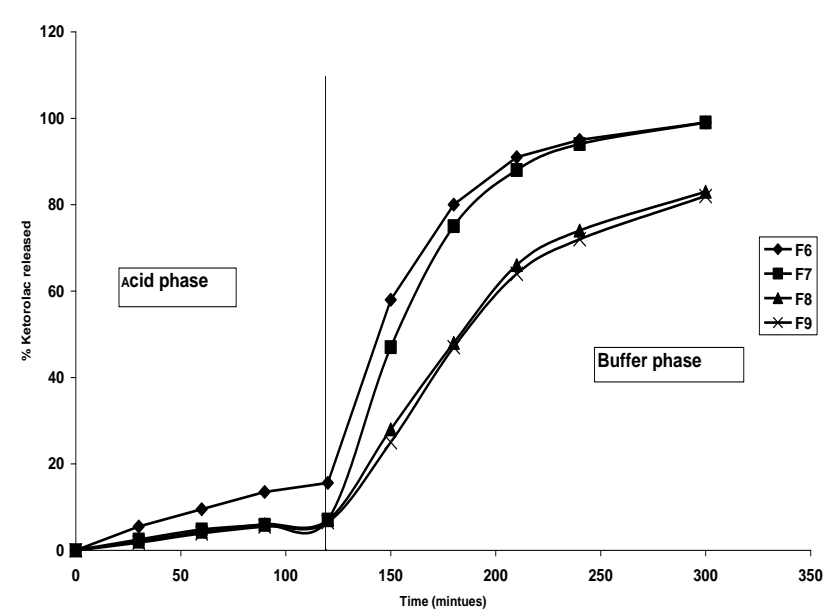

Fig. 7: Ketorolac release profiles from Eudragit L100-55 pellets in $0.1 \mathrm{~N} \mathrm{HCl}$, then in phosphate buffer at $\mathrm{pH} 6.8$, at $37^{\circ} \mathrm{C}$.

\section{Conclusion}

Enteric matrix pellets with a diameter below $2 \mathrm{~mm}$ and containing 5\% ketorolac could be successfully prepared by extrusion/spheronization. Both types of Eudragit employed were suitable as matrix forming agent and allowed preparation of enteric matrix pellets. Addition of Avicel is essential as it aids the extrusion process and increases the gastric resistance of pellets to be within the limits of USP. EUL formulae gave higher release rate in phosphate buffer phase while their release in acidic medium is very low, so it is more suitable for use as enteric matrix pellets; while EUS containing formulations gave more sustained drug release in the phosphate buffer phase.

\section{REFERENCES}

1- A. Sandberg, G. Ragnarsson, U. E. Jonsson and J. Sjogren, "Design of a new multiple-unit controlled-release formulation of metoprolol-metoprolol CR", Eur. J. Clin. Pharmacol., 33, S3 (1988).

2- W. G. Chambliss, in: I. Ghebre-Sellassie (Ed), "Pharmaceutical Pelletization Technology," Marcel Dekker, 1989, p.15.

3- H. Bechgaard and G. H. Nielsen, "Controlled-release multiple-units and single-unit doses. A literature review", Drug Dev. Ind. Pharm., 4, 53 (1978).

4- R. L. Robinson and R. G. Hollenbeck, "Manufacture of spherical acetaminophen pellets: comparison of rotary processing with multi-step extrusion and spheronization", Pharm. Technol., 15, 48 (1991).

5- S. U. Schilling, N. H. Shah, A. W. Malick and J. W. McGinity, "Properties of melt extruded enteric matrix pellets", Eur. J. Pharm. Biopharm., 74, 352 (2010).

6- G. P. Andrews, D. S. Jones, O. A. Diak, C. P. McCoy, A. B. Watts and J. W. McGinity, "The manufacture and characterization of hot-melt extruded enteric tablets", ibid., 69, 264 (2008).

7- R. Yang, Y. Wang, X. Zheng, J. Meng, X. Tang, $X$. Zhang, "Preparation and evaluation of ketoprofen hot-melt extruded enteric and sustained-release tablets", Drug Dev. Ind. Pharm., 34, 83 (2008).

8- D. Bruce, N. H. Shah, A. W. Malick, M. H. Infeld and J. W. McGinity, "Properties of hot-melt extruded tablet formulation for colonic delivery of 5-aminosalicylic acid", Eur. J. Pharm. Biopharm., 59, 85 (2005).

9- C. Vervaet, L. Baert and J. P. Remon, "Extrusion-spheronisation. A literature review", Int. J. Pharm., 116, 131 (1995).

10- P. B. Deasy and M. F. L. Law, "Use of extrusion- spheronization to develop an improved dosage form of indomethacin", ibid., 148, 201 (1997).

11- M. D. Parker, R. C. Rowe and N. G. Upjohn, "Mixer torque rheometry: A method for quantifying the consistency of wet granulations", Pharm. Tech. Int., 2, 50 (1990). 
12- F. Khan and N. Pilpel, "The effect of particle size and moisture on the tensile strength of microcrystalline cellulose powder", Powder Technol., 48, 145 (1986).

13- K. E. Fielden, J. M. Newton, P. O’Brien and R. C. Rowe, "Thermal studies on the interaction of water and microcrystalline cellulose", J. Pharm. Pharmacol., 40, 674 (1988).

14- D. Sixsmith, "The effect of compression on some physical properties of microcrystalline cellulose powders", ibid., 29, 33 (1977).

15- R. Chatlapalli and B. D. Rohera, "Rheological characterization of diltiazem $\mathrm{HCl}$ : cellulose wet masses using a mixer torque Rheometer", Int. J. Pharm., 175, 47 (1998).

16- K. A. Mehta, M. S. Kislalioglu, W. Phuapradit, A. W. Malick and N. H. shah, "Release performance of a poorly soluble drug from a novel, Eudragit $\AA$ - based multi-unit erosion matrix", ibid., 213, 7 (2001).
17- M. F. L. Law and P. B. Deasy, "Use of hydrophilic polymers with microcrystalline cellulose to improve extrusion- spheronization", Eur. J. Pharm. Biopharm., 45, 57-65 (1998).

18- United States Pharmacopeia $24^{\text {th }}$ Ed. \& National Formulary The United States Pharmacopeial Convention Inc. Rockville, MD., 2000, p. 1947.

19- M. R. Abbaspour, F. Sadeghi and H. Afrasiabi Garekani, "Preparation and characterization of ibuprofen pellets based on Eudragit RS PO and RL PO or their combination", Int. J. Pharm., 303, 88-94 (2005).

20- G. M. Mahrous, M. A. Ibarhim, M. ElBadry and F. K. Al-Anazi, "Indomethacin sustained release pellets prepared by extrusion / spheronization", J. Drug Del. Tech., 20, 119 (2010). 